\title{
DER KORAN
}

Arabisch-Deutsch 


\title{
DER KORAN
}

Arabisch-Deutsch

\author{
Übersetzung \\ und wissenschaftlicher Kommentar \\ von \\ Adel Theodor Khoury
}

Band 3

Gütersloher Verlagshaus 


\title{
DER KORAN
}

Arabisch-Deutsch

\author{
Übersetzung \\ und wissenschaftlicher Kommentar \\ von \\ Adel Theodor Khoury \\ Band 3
}

Sure 2,213-2,286

1992

Gütersloher Verlagshaus 
Bibliografische Information der Deutschen Nationalbibliothek Die Deutsche Nationalbibliothek verzeichnet diese Publikation in der Deutschen Nationalbibliografie; detaillierte bibliografische

Daten sind im Internet über https://portal.dnb.de abrufbar.

Copyright @ 1992 Gütersloher Verlagshaus, Gütersloh, in der Verlagsgruppe Random House GmbH, Neumarkter Str. 28, 81673 München

Der Inhalt dieses E-Books ist urheberrechtlich geschützt und enthält technische Sicherungsmaßnahmen gegen unbefugte Nutzung.

Die Entfernung dieser Sicherung sowie die Nutzung durch unbefugte Verarbeitung, Vervielfältigung, Verbreitung oder öffentliche Zugänglichmachung, insbesondere in elektronischer Form, ist untersagt und kann straf- und zivilrechtliche Sanktionen nach sich ziehen.

Sollte diese Publikation Links auf Webseiten Dritter enthalten, so übernehmen wir für deren Inhalte keine Haftung, da wir uns diese nicht zu eigen machen, sondern lediglich auf deren Stand zum Zeitpunkt der Erstveröffentlichung verweisen.

Umschlaggestaltung: Dieter Rehder, B-Kelmis

Gesamtherstellung: ICS Communikations-Service GmbH, Bergisch Gladbach ISBN 978-3-641-24750-8

www.gtvh.de 